\title{
A Mathematical Analysis of the Complex Dipole Simulation Method
}

\author{
Koya SAKAKIBARA and Masashi KATSURADA
}

The University of Tokyo and Meiji University

\begin{abstract}
We propose the complex dipole simulation method (CDSM) which approximates a holomorphic function by linear combination of $1 /(z-\zeta)$ with the use of its boundary values. In this paper, we treat a function $f$ which is holomorphic in $\Omega$ and continuous on $\bar{\Omega}$ in the case where $\Omega$ is a disk or the exterior domain of a disk. Then we establish the following fact: if $f$ is holomorphic in some neighborhood of $\bar{\Omega}$, the error of an approximate function $f^{(N)}$ decays exponentially with respect to $N$, where $N$ is the number of the charge points.
\end{abstract}

\section{Introduction}

The objectives of this paper are to propose the complex dipole simulation method (CDSM) which is an approximation technique for a holomorphic function $f$ by using its boundary values, to prove the unique existence of the approximate function $f^{(N)}$ and the exponential decay of the error of $f^{(N)}$, and to exemplify the effectiveness of CDSM by numerical experiments.

To begin with, we explain the dipole simulation method (DSM) which is one branch of the charge simulation method (CSM). Let us consider the Laplace equation with the Dirichlet boundary condition:

$$
\begin{aligned}
\triangle u=0 & \text { in } \Omega, \\
u=\phi & \text { on } \partial \Omega,
\end{aligned}
$$

where $\Omega$ is a bounded domain in $\mathbb{R}^{2}$ with a smooth boundary $\partial \Omega$. $\phi$ is a given function defined on $\partial \Omega$. DSM gives an approximate solution $u_{\mathrm{D}}^{(N)}$ for the potential problem (1.1)(1.2) of the form

$$
u_{\mathrm{D}}^{(N)}(\boldsymbol{x})=\sum_{k=1}^{N} Q_{k} \frac{-1}{2 \pi} \frac{\left(\boldsymbol{n}_{k} \mid \boldsymbol{x}-\boldsymbol{\xi}_{k}\right)}{\left\|\boldsymbol{x}-\boldsymbol{\xi}_{k}\right\|^{2}}
$$

Received February 3, 2014; revised February 26, 2015

Mathematics Subject Classification: 32A10, 41A20, 30E10, 65N35, 65N12

Key words and phrases: Charge Simulation Method, Method of Fundamental Solutions, Rational Function Approximation 
where $\left\{\boldsymbol{\xi}_{k}\right\}_{k=1}^{N}$ are taken from the exterior of $\Omega, \boldsymbol{n}_{k}$ is a unit vector which represents the direction of the axis of the dipole at $\xi_{k},(\cdot \mid \cdot)$ denotes the two-dimensional Euclidean inner product, $\|\cdot\|$ is the two-dimensional Euclidean norm defined by $\|\cdot\|=\sqrt{(\cdot \mid \cdot)}$ and the real coefficients $\left\{Q_{k}\right\}_{k=1}^{N}$ are determined by the collocation method. Namely, we take $N$ points $\left\{\boldsymbol{x}_{j}\right\}_{j=1}^{N}$ on $\partial \Omega$ and determine $\left\{Q_{k}\right\}_{k=1}^{N}$ by the equations below:

$$
u_{\mathrm{D}}^{(N)}\left(\boldsymbol{x}_{j}\right)=\phi\left(\boldsymbol{x}_{j}\right) \quad(j=1,2, \ldots, N) .
$$

Katsurada [3] studied DSM in the case where $\Omega$ is a two-dimensional disk $D_{\rho}=\{\boldsymbol{x} \in$ $\left.\mathbb{R}^{2} ;\|\boldsymbol{x}\|<\rho\right\}(\rho>0)$. In [3], $\left\{\boldsymbol{x}_{j}\right\}_{j=1}^{N}$ and $\left\{\boldsymbol{\xi}_{k}\right\}_{k=1}^{N}$ are located at $\left(\rho \cos \theta_{j}, \rho \sin \theta_{j}\right)(j=$ $1,2, \ldots, N)$ and $\left(R \cos \theta_{k}, R \sin \theta_{k}\right)(k=1,2, \ldots, N)$, respectively, where $\theta_{j}=2 \pi j / N$ and $R \in] \rho,+\infty$ [, and $\boldsymbol{n}_{k}$ 's are settled as $\boldsymbol{n}_{k}=-(1 / R) \boldsymbol{\xi}_{k}$. In this situation, it is proved the unique existence of the approximate solution $u_{\mathrm{D}}^{(N)}$ and the exponential decay of the error of $u_{\mathrm{D}}^{(N)}$. The convergence theorem in [3] is as follows:

THEOREM 1.1 ([3]). Suppose that the boundary data $\phi$ is real analytic. In this case the exact solution $u$ for the potential problem (1.1)-(1.2) admits a harmonic extension to some neighborhood of $\bar{\Omega}$. Hence we may assume that $u$ is harmonic in $D_{r_{0}}$ and continuous on $\bar{D}_{r_{0}}$ with $\left.r_{0} \in\right] \rho,+\infty[$. Then there exists a positive constant $C$, which is independent of $N$, such that

$$
\sup _{\boldsymbol{x} \in \Omega}\left|u(\boldsymbol{x})-u_{\mathrm{D}}^{(N)}(\boldsymbol{x})\right| \leq C \times \begin{cases}\left(\frac{\rho}{R}\right)^{N} & \text { if } \frac{\rho}{R}>\sqrt{\frac{\rho}{r_{0}}}, \\ N\left(\frac{\rho}{r_{0}}\right)^{N / 2} & \text { if } \frac{\rho}{R}=\sqrt{\frac{\rho}{r_{0}}} \\ \left(\frac{\rho}{r_{0}}\right)^{N / 2} & \text { if } \frac{\rho}{R}<\sqrt{\frac{\rho}{r_{0}}} .\end{cases}
$$

The corresponding results for CSM are proved in Katsurada and Okamoto [2] and [3].

On the other hand, Ogata [6] proposed a generalized DSM (GDSM) in a sense that $\left\{\boldsymbol{n}_{k}\right\}_{k=1}^{N}$ are also unknown. Namely GDSM offers an approximate solution of the form

$$
u_{\mathrm{GD}}^{(N)}(\boldsymbol{x})=\sum_{k=1}^{N} \frac{-1}{2 \pi} \frac{\left(\boldsymbol{p}_{k} \mid \boldsymbol{x}-\boldsymbol{\xi}_{k}\right)}{\left\|\boldsymbol{x}-\boldsymbol{\xi}_{k}\right\|^{2}},
$$

where $\left\{\boldsymbol{\xi}_{k}\right\}_{k=1}^{N}$ are chosen from the exterior of $\Omega$ and the two-dimensional real coefficient vector $\left\{\boldsymbol{p}_{k}\right\}_{k=1}^{N}$ are determined by the collocation method. Since the degree of freedom of GDSM is twice as that of DSM, we take $2 N$ points $\left\{\boldsymbol{x}_{j}\right\}_{j=1}^{2 N}$ on $\partial \Omega$ and determine $\left\{\boldsymbol{p}_{k}\right\}_{k=1}^{N}$ by the equations below:

$$
u_{\mathrm{GD}}^{(N)}\left(\boldsymbol{x}_{j}\right)=f\left(\boldsymbol{x}_{j}\right) \quad(j=1,2, \ldots, 2 N) .
$$


Ogata also extended the original DSM proposed by Katsurada to Jordan regions and examined its convergence numerically [7].

At this point, we rewrite the GDSM and DSM approximate solutions in terms of the real part of the complex function:

$$
u_{\mathrm{GD}}^{(N)}(\boldsymbol{x})=u_{\mathrm{D}}^{(N)}(\boldsymbol{x})=\operatorname{Re}\left(\sum_{k=1}^{N} \frac{-1}{2 \pi} \frac{Q_{k}}{z-\zeta_{k}}\right),
$$

where

$$
Q_{k}=p_{k}+\mathrm{i} q_{k}, \quad z=x+\mathrm{i} y, \quad \zeta_{k}=\xi_{k}+\mathrm{i} \eta_{k}
$$

in which

$$
\boldsymbol{p}_{k}=Q_{k} \boldsymbol{n}_{k}=\left(p_{k}, q_{k}\right), \quad \boldsymbol{x}=(x, y), \quad \boldsymbol{\xi}_{k}=\left(\xi_{k}, \eta_{k}\right) .
$$

In view of this expression, we propose CDSM for approximating holomorphic functions. In detail, let $\Omega$ be a domain in the complex plane and $f$ be a function which is holomorphic in $\Omega$ and continuous on $\bar{\Omega}$. CDSM gives an approximate function for $f$ as

$$
f^{(N)}(z)=\sum_{k=1}^{N} \frac{Q_{k}}{z-\zeta_{k}}
$$

where $\left\{\zeta_{k}\right\}_{k=1}^{N}$ are taken from the exterior of $\Omega$ and the complex coefficients $\left\{Q_{k}\right\}_{k=1}^{N}$ are determined by the collocation method, that is, we take $N$ points $\left\{z_{j}\right\}_{j=1}^{N}$ on $\partial \Omega$ and determine $\left\{Q_{k}\right\}_{k=1}^{N}$ by the equations below:

$$
f^{(N)}\left(z_{j}\right)=f\left(z_{j}\right) \quad(j=1,2, \ldots, N) .
$$

This is a description of CDSM. We call $\left\{z_{j}\right\}_{j=1}^{N},\left\{\zeta_{k}\right\}_{k=1}^{N}$ and the equations (1.5) the collocation points, the charge points and the collocation equations, respectively.

The readers may think that a holomorphic function can be approximated by DSM by considering its real part and its imaginary part separately. Indeed, when we write the boundary values of $f=u+\mathrm{i} v$ as $\left.f\right|_{\partial \Omega}=\phi+\mathrm{i} \psi$, then $u$ and $v$ are characterized by two Dirichlet problems:

$$
\begin{aligned}
& \triangle u=0 \quad \text { in } \Omega, \quad u=\phi \quad \text { on } \partial \Omega, \\
& \Delta v=0 \quad \text { in } \Omega, \quad v=\psi \quad \text { on } \partial \Omega \text {. }
\end{aligned}
$$

Although the collocation equations

$$
f^{(N)}\left(z_{j}\right)=f\left(z_{j}\right) \quad(j=1,2, \ldots, N)
$$

are solvable, the collocation equations

$$
\operatorname{Re} f^{(N)}\left(z_{j}\right)=u_{\mathrm{GD}}^{(N)}\left(z_{j}\right)=f\left(z_{j}\right) \quad(j=1,2, \ldots, N)
$$


are not solvable since the degree of freedom of GDSM is twice as that of CDSM. Furthermore, since the error of CDSM's approximate function $f^{(N)}$ is evaluated as

$$
\sup _{z \in \bar{\Omega}}\left|f(z)-f^{(N)}(z)\right| \leq C\left(\frac{\rho}{R}\right)^{N}+C^{\prime}\left(\frac{\rho}{r_{0}}\right)^{N},
$$

(we will show details in Theorems 2.2 and 2.4), where $C$ and $C^{\prime}$ are constants independent of $N$, comparing (1.3) with (1.6), we see that the convergence rates are different from each other. Therefore the theory of CDSM are not followed from the one of DSM.

This paper consists of six sections. In Section 2, we state theorems on the convergence and error estimates and we prove them in Section 3. In Section 4, we calculate the condition number of the coefficient matrix for the linear system (1.5) explicitly. In Section 5, we show some numerical experiments in the case where $\Omega$ is a disk, and moreover we compare the results for CDSM with DSM. In Section 6, we summarize this paper and give some concluding remarks.

\section{Main Results}

We prepare some notations beforehand.

NotATions 1. For a positive number $r$, we define

$$
\begin{aligned}
D_{r} & :=\{z \in \mathbb{C} ;|z|<r\}, \\
\gamma_{r} & :=\partial D_{r}=\{z \in \mathbb{C} ;|z|=r\}, \\
D_{r}^{*} & :=\mathbb{C} \backslash \bar{D}_{r}=\{z \in \mathbb{C} ;|z|>r\} .
\end{aligned}
$$

Firstly we consider the case $\Omega=D_{\rho}$, where $\rho$ is a positive number. The collocation points $\left\{z_{j}\right\}_{j=1}^{N}$ and the charge points $\left\{\zeta_{k}\right\}_{k=1}^{N}$ are located at $\rho \omega^{j-1}(j=1,2, \ldots, N)$ and $R \omega^{k-1}(k=1,2, \ldots, N)$, respectively, where $\left.R \in\right] \rho,+\infty[$ and $\omega=\exp (2 \pi \mathrm{i} / N)$. We assume that the function $f$ is holomorphic in $\Omega$ and continuous on $\bar{\Omega}$.

We seek the approximate function $f^{(N)}$ within the function space $\mathscr{X}^{(N)}$ which is defined as follows:

$$
\mathscr{X}^{(N)}=\mathscr{X}^{(N)}\left(\left\{\zeta_{k}\right\}_{k=1}^{N}\right)=\left\{\sum_{k=1}^{N} Q_{k} H\left(\cdot, \zeta_{k}\right) ;\left(Q_{1}, Q_{2}, \ldots, Q_{N}\right)^{\mathrm{T}} \in \mathbb{C}^{N}\right\},
$$

where

$$
H(z, \zeta)=\frac{1}{z-\zeta}
$$


We introduce a norm $\|\cdot\|_{r}: X \rightarrow \mathbb{R}$ for each $\left.r \in\right] 0,+\infty[$ as follows:

$$
\|f\|_{r}=\sum_{n=0}^{\infty}\left|a_{n}\right| r^{n}
$$

where $X$ is a function space whose elements are holomorphic functions in $D_{\rho}$ and

$$
f(z)=\sum_{n=0}^{\infty} a_{n} z^{n} \quad\left(f \in X ; z \in D_{\rho}\right) .
$$

We are now in a position to state the theorems:

THEOREM 2.1. We can determine $f^{(N)} \in \mathscr{X}^{(N)}$ by (1.5) uniquely.

THEOREM 2.2. Assume that $f$ is holomorphic in some neighborhood of $\bar{\Omega}$. If we choose $\left.r_{0} \in\right] \rho,+\infty$ [ so that $\|f\|_{r_{0}}<+\infty$, then we have the inequality

$$
\sup _{z \in \bar{\Omega}}\left|f(z)-f^{(N)}(z)\right| \leq \frac{2\|f\|_{\rho}}{1-(\rho / R)^{N}}\left(\frac{\rho}{R}\right)^{N}+2\|f\|_{r_{0}}\left(\frac{\rho}{r_{0}}\right)^{N}
$$

for the approximate function $f^{(N)}$ in Theorem 2.1.

Secondly we consider the case $\Omega=D_{\rho}^{*}$, where $\rho$ is a positive number. We take the collocation points $\left\{z_{j}\right\}_{j=1}^{N}$ and the charge points $\left\{\zeta_{k}\right\}_{k=1}^{N}$ similarly in the case $\Omega=D_{\rho}$, where $R \in] 0, \rho$ [. We assume that the function $f$ is holomorphic and bounded in $\Omega$ and continuous on $\bar{\Omega}$.

We seek the approximate function $f^{(N)}$ within the function space $\mathscr{Y}^{(N)}$ which is defined as follows:

$$
\mathscr{Y}^{(N)}=\mathscr{Y}^{(N)}\left(\left\{\zeta_{k}\right\}_{k=1}^{N}\right)=\left\{\sum_{k=1}^{N} Q_{k} \Gamma\left(\cdot, \zeta_{k}\right) ;\left(Q_{1}, Q_{2}, \ldots, Q_{N}\right)^{\mathrm{T}} \in \mathbb{C}^{N}\right\},
$$

where

$$
\Gamma(z, \zeta)=\frac{z}{z-\zeta}
$$

REMARK 1. We cannot use $H(\cdot, \cdot)$ as a substitute for $\Gamma(\cdot, \cdot)$ in $\mathscr{Y}^{(N)}$ since if we use $H(\cdot, \cdot)$, then the value at the point at infinity becomes zero. Inversely we cannot use $\Gamma(\cdot, \cdot)$ instead of $H(\cdot, \cdot)$ in $\mathscr{X}^{(N)}$ since if we use $\Gamma(\cdot, \cdot)$, then the value at the origin becomes zero. However, we can use $\mathscr{H}(z, \zeta)=\zeta /(z-\zeta)$ in place of $H(z, \zeta)$ in $\mathscr{X}^{(N)}$ and obtain similar theorems with Theorems 2.1 and 2.2. 
We introduce the norm $\|\cdot\|_{r}: Y \rightarrow \mathbb{R}$ for each $\left.r \in\right] 0,+\infty[$ as follows:

$$
\|f\|_{r}=\sum_{n=0}^{\infty}\left|b_{-n}\right| r^{-n},
$$

where $Y$ is a function space whose elements are holomorphic and bounded in $\Omega$ and

$$
f(z)=\sum_{n=0}^{\infty} b_{-n} z^{-n} \quad\left(f \in Y ; z \in D_{\rho}^{*}\right) .
$$

We can state the theorems:

THEOREM 2.3. We can determine $f^{(N)} \in Y^{(N)}$ by (1.5) uniquely.

THEOREM 2.4. Assume that $f$ is holomorphic in some neighborhood of $\bar{\Omega}$. If we choose $\left.r_{0} \in\right] 0, \rho\left[\right.$ so that $\|f\|_{r_{0}}<+\infty$, then we have the inequality

$$
\sup _{z \in \bar{\Omega}}\left|f(z)-f^{(N)}(z)\right| \leq \frac{2\|f\|_{\rho}}{1-(R / \rho)^{N}}\left(\frac{R}{\rho}\right)^{N}+2\|f\|_{r_{0}}\left(\frac{r_{0}}{\rho}\right)^{N}
$$

for the approximate function $f^{(N)}$ in Theorem 2.3.

\section{Proofs of Theorems}

The proofs of Theorems 2.1, 2.2, 2.3 and 2.4 are given in this section. The proof techniques employed here are based on the ones due to [2].

We first prove Theorem 2.1. We rewrite the collocation equations (1.5) as follows:

$$
\sum_{k=1}^{N} \frac{1}{\omega^{j-1}} \frac{Q_{k}}{\rho-R \omega^{k-j}}=f\left(z_{j}\right), \quad j=1,2, \ldots, N,
$$

therefore (1.5) is equivalent to the linear system

$$
G Q=f,
$$

where $\boldsymbol{Q}=\left(Q_{1}, Q_{2}, \ldots, Q_{N}\right)^{\mathrm{T}} \in \mathbb{C}^{N}, \boldsymbol{f}=\left(f\left(z_{1}\right), \omega f\left(z_{2}\right), \ldots, \omega^{N-1} f\left(z_{N}\right)\right)^{\mathrm{T}} \in \mathbb{C}^{N}$ and $G$ is an $N \times N$ complex matrix with the entries $g_{j k}=1 /\left(\rho-R \omega^{k-j}\right)(j, k=1,2, \ldots, N)$. Let $W=\left[W_{j k} ; j, k=1,2, \ldots, N\right]$ be the $N$-dimensional discrete Fourier transform, that is,

$$
W_{j k}=\frac{1}{\sqrt{N}} \omega^{(j-1)(k-1)} \quad(j, k=1,2, \ldots, N) .
$$

We note that $G$ is a circulant matrix whose $(j, k)$ entry depends on $k-j(\bmod N)$. In general, circulant matrices can be diagonalized by the discrete Fourier transform, indeed we see

$$
W^{-1} G W=\operatorname{diag}\left[\varphi_{0}^{(N)}(\rho), \varphi_{1}^{(N)}(\rho), \ldots, \varphi_{N-1}^{(N)}(\rho)\right]
$$


where

$$
\varphi_{p}^{(N)}(z)=\sum_{k=1}^{N} \omega^{p(k-1)} H\left(z, \zeta_{k}\right) \quad\left(z \in \mathbb{C} \backslash\left\{\zeta_{k}\right\}_{k=1}^{N} ; p \in \mathbb{Z}\right) .
$$

The function $\varphi_{p}^{(N)}$ plays a fundamental role in the following analysis. We first prove the following:

LEMMA 3.1. (i) $\varphi_{p}^{(N)}$ is periodic with respect to $p$ with period $N$.

(ii) We have

$$
\varphi_{p}^{(N)}(z)=-\frac{N}{R}\left(\frac{z}{R}\right)^{p-1} \frac{1}{1-(z / R)^{N}} \quad\left(z \in D_{R}\right)
$$

for all $p \in\{1,2, \ldots, N\}$.

Proof. (i) $\varphi_{p}^{(N)}=\varphi_{q}^{(N)}$ follows from $(3.3)$ if $p \equiv q(\bmod N)$ since $\omega^{N}=1$.

(ii) To begin with, we expand $H\left(z, \zeta_{k}\right)$ as

$$
H\left(z, \zeta_{k}\right)=\frac{-1}{R \omega^{k-1}} \frac{1}{1-z /\left(R \omega^{k-1}\right)}=-\frac{1}{R} \sum_{n=0}^{\infty}\left(\frac{z}{R}\right)^{n} \omega^{-(k-1)(n+1)} \quad\left(z \in D_{R}\right) .
$$

Hence we have

$$
\begin{aligned}
\varphi_{p}^{(N)}(z) & =\sum_{k=1}^{N} \omega^{p(k-1)} H\left(z, \zeta_{k}\right)=\sum_{k=1}^{N} \omega^{p(k-1)}\left[-\frac{1}{R} \sum_{n=0}^{\infty}\left(\frac{z}{R}\right)^{n} \omega^{-(k-1)(n+1)}\right] \\
& =-\frac{1}{R} \sum_{n=0}^{\infty}\left(\frac{z}{R}\right)^{n} \sum_{k=1}^{N} \omega^{-(n-(p-1))(k-1)}=-\frac{N}{R} \sum_{n \equiv p-1}\left(\frac{z}{n \geq 0}\right)^{n}(\bmod N) \\
& =-\frac{N}{R}\left(\frac{z}{R}\right)^{p-1} \frac{1}{1-(z / R)^{N}}
\end{aligned}
$$

for all $p \in\{1,2, \ldots, N\}$.

Proof of Theorem 2.1. By (3.2) and Lemma 3.1 (i), we can compute the determinant of $G$ as

$$
\operatorname{det} G=\prod_{p=0}^{N-1} \varphi_{p}^{(N)}(\rho)=\prod_{p=1}^{N} \varphi_{p}^{(N)}(\rho)
$$

By Lemma 3.1 (ii), we know $\varphi_{p}^{(N)}(\rho)<0(p=1,2, \ldots, N)$. Hence $\operatorname{det} G \neq 0$ follows and this completes the proof of Theorem 2.1. 
By (3.2), the inverse matrix of $G$ is as follows:

$$
G^{-1}=W \operatorname{diag}\left[\frac{1}{\varphi_{0}^{(N)}(\rho)}, \frac{1}{\varphi_{1}^{(N)}(\rho)}, \ldots, \frac{1}{\varphi_{N-1}^{(N)}(\rho)}\right] W^{-1} .
$$

A direct calculation yields

$$
\left[G^{-1}\right]_{k j}=\frac{1}{N} \sum_{p=1}^{N} \frac{\omega^{(k-j)(p-1)}}{\varphi_{p-1}^{(N)}(\rho)} \quad(j, k=1,2, \ldots, N),
$$

where $\left[G^{-1}\right]_{k j}$ denotes the $(k, j)$ element of $G^{-1}$. This makes it possible to solve (3.1) as

$$
\begin{aligned}
Q_{k} & =\sum_{j=1}^{N}\left[G^{-1}\right]_{k j}[\boldsymbol{f}]_{j}=\sum_{j=1}^{N}\left(\frac{1}{N} \sum_{p=1}^{N} \frac{\omega^{(k-j)(p-1)}}{\varphi_{p-1}^{(N)}(\rho)}\right) \omega^{j-1} f\left(z_{j}\right) \\
& =\frac{1}{N} \sum_{j, p=1}^{N} \frac{\omega^{(k-1)(p-1)-(j-1)(p-2)}}{\varphi_{p-1}^{(N)}(\rho)} f\left(z_{j}\right),
\end{aligned}
$$

hence we have

$$
\begin{aligned}
f^{(N)}(z) & =\sum_{k=1}^{N} Q_{k} H\left(z, \zeta_{k}\right)=\sum_{k=1}^{N}\left(\frac{1}{N} \sum_{j, p=1}^{N} \frac{\omega^{(k-1)(p-1)-(j-1)(p-2)}}{\varphi_{p-1}^{(N)}(\rho)} f\left(z_{j}\right)\right) H\left(z, \zeta_{k}\right) \\
& =\frac{1}{N} \sum_{j, p=1}^{N} \omega^{-(j-1)(p-2)} \frac{\varphi_{p-1}^{(N)}(z)}{\varphi_{p-1}^{(N)}(\rho)} \sum_{n=0}^{\infty} a_{n} \rho^{n} \omega^{(j-1) n} \\
& =\frac{1}{N} \sum_{p=1}^{N} \frac{\varphi_{p-1}^{(N)}(z)}{\varphi_{p-1}^{(N)}(\rho)} \sum_{n=0}^{\infty} a_{n} \rho^{n}\left(\sum_{j=1}^{N} \omega^{(n-(p-2))(j-1)}\right) \\
& =\sum_{p=1}^{N} \frac{\varphi_{p-1}^{(N)}(z)}{\varphi_{p-1}^{(N)}(\rho)} \sum_{n \equiv p-2} a_{n \geq 0} a_{n} \rho^{n}=\sum_{n=0}^{\infty} a_{n} \rho^{n} \frac{\varphi_{n+1}^{(N)}(z)}{\varphi_{n+1}^{(N)}(\rho)} .
\end{aligned}
$$

Consequently we can write the error function $e^{(N)}=f-f^{(N)}$ as

$$
e^{(N)}(z)=\sum_{n=0}^{\infty} a_{n} \rho^{n}\left[\left(\frac{z}{\rho}\right)^{n}-\frac{\varphi_{n+1}^{(N)}(z)}{\varphi_{n+1}^{(N)}(\rho)}\right] .
$$

Since $e^{(N)}$ is holomorphic in $\Omega$ and continuous on $\bar{\Omega}$, we obtain by the maximum modulus principle for holomorphic functions

$$
\sup _{z \in \bar{\Omega}}\left|e^{(N)}(z)\right| \leq \sum_{n=0}^{\infty}\left|a_{n}\right| \rho^{n} g_{n, \rho}^{(N)},
$$


where

$$
g_{n, \rho}^{(N)}:=\sup _{z \in \gamma_{\rho}}\left|\left(\frac{z}{\rho}\right)^{n}-\frac{\varphi_{n+1}^{(N)}(z)}{\varphi_{n+1}^{(N)}(\rho)}\right| .
$$

At this stage, we claim the following Lemma 3.2 concerning $g_{n, \rho}^{(N)}$.

LEMMA 3.2. (i) For all $n \in \mathbb{N} \cup\{0\}$, we have

$$
g_{n, \rho}^{(N)} \leq 2 .
$$

(ii) For all $p \in\{0,1, \ldots, N-1\}$, we have

$$
g_{p, \rho}^{(N)} \leq \frac{2(\rho / R)^{N}}{1-(\rho / R)^{N}} .
$$

PROOF. (i) We know by Lemma 3.1 that the inequality

$$
\left|\varphi_{p}^{(N)}(z)\right| \leq\left|\varphi_{p}^{(N)}(|z|)\right|
$$

holds for all $p \in \mathbb{Z}$ and for all $z \in D_{R}$. Therefore we see

$$
\left|\left(\frac{z}{\rho}\right)^{n}-\frac{\varphi_{n+1}^{(N)}(z)}{\varphi_{n+1}^{(N)}(\rho)}\right| \leq\left(\frac{|z|}{\rho}\right)^{n}+\frac{\left|\varphi_{n+1}^{(N)}(z)\right|}{\left|\varphi_{n+1}^{(N)}(\rho)\right|} \leq 2
$$

for all $z \in \gamma_{\rho}$. This establishes (3.5).

(ii) By Lemma 3.1, we have

$$
\left|\left(\frac{z}{\rho}\right)^{p}-\frac{\varphi_{p+1}^{(N)}(z)}{\varphi_{p+1}^{(N)}(\rho)}\right|=\left|\left(\frac{z}{\rho}\right)^{p} \frac{(\rho / R)^{N}-(z / R)^{N}}{1-(z / R)^{N}}\right| \leq \frac{2(\rho / R)^{N}}{1-(\rho / R)^{N}}
$$

for all $z \in \gamma_{\rho}$. Thus (3.6) is shown and this completes the proof of Lemma 3.2.

Proof OF THEOREM 2.2. By (3.4) and Lemma 3.2, we have

$$
\begin{aligned}
\sup _{z \in \bar{\Omega}}\left|f(z)-f^{(N)}(z)\right| & \leq\left[\sum_{n=0}^{N-1}+\sum_{n=N}^{\infty}\right]\left|a_{n}\right| \rho^{n} g_{n, \rho}^{(N)} \\
& \leq \sum_{n=0}^{N-1}\left|a_{n}\right| \rho^{n} \frac{2(\rho / R)^{N}}{1-(\rho / R)^{N}}+\sum_{n=N}^{\infty}\left|a_{n}\right| \rho^{n} \cdot 2 \\
& \leq \sum_{n=0}^{\infty}\left|a_{n}\right| \rho^{n} \frac{2(\rho / R)^{N}}{1-(\rho / R)^{N}}+2 \sum_{n=N}^{\infty}\left|a_{n}\right| r_{0}^{n}\left(\frac{\rho}{r_{0}}\right)^{n} \\
& \leq \frac{2\|f\|_{\rho}}{1-(\rho / R)^{N}}\left(\frac{\rho}{R}\right)^{N}+2\|f\|_{r_{0}}\left(\frac{\rho}{r_{0}}\right)^{N} .
\end{aligned}
$$


Theorems 2.3 and 2.4 are proved as well as Theorems 2.1 and 2.2, respectively, therefore we only sketch out the proofs of them.

We rewrite the collocation equations (1.5) into (3.1) similarly, where $\boldsymbol{Q}=$ $\left(Q_{1}, Q_{2}, \ldots, Q_{N}\right)^{\mathrm{T}} \in \mathbb{C}^{N}, \boldsymbol{f}=\left(f\left(z_{1}\right), f\left(z_{2}\right), \ldots, f\left(z_{N}\right)\right)^{\mathrm{T}} \in \mathbb{C}^{N}$ and $G$ is an $N \times N$ complex matrix with the entries $g_{j k}=\Gamma\left(z_{j}, \zeta_{k}\right)(j, k=1,2, \ldots, N)$. $G$ is a circulant matrix whose $(j, k)$ entry depends on $k-j(\bmod N)$, therefore we see

$$
W^{-1} G W=\operatorname{diag}\left[\psi_{0}^{(N)}(\rho), \psi_{1}^{(N)}(\rho), \ldots, \psi_{N-1}^{(N)}(\rho)\right],
$$

where

$$
\psi_{p}^{(N)}(z):=\sum_{k=1}^{N} \omega^{p(k-1)} \Gamma\left(z, \zeta_{k}\right) \quad\left(z \in \mathbb{C} \backslash\left\{\zeta_{k}\right\}_{k=1}^{N} ; p \in \mathbb{Z}\right)
$$

We have the following Lemma 3.3 as to the function $\psi_{p}^{(N)}$.

LEMMA 3.3. (i) $\psi_{p}^{(N)}$ is periodic with respect to $p$ with period $N$.

(ii) We have

$$
\psi_{p}^{(N)}(z)=N\left(\frac{R}{z}\right)^{N-p} \frac{1}{1-(R / z)^{N}} \quad\left(z \in D_{R}^{*}\right)
$$

for all $p \in\{1,2, \ldots, N\}$.

By (3.7), $G^{-1}$ is as follows:

$$
G^{-1}=W \operatorname{diag}\left[\frac{1}{\psi_{0}^{(N)}(\rho)}, \frac{1}{\psi_{1}^{(N)}(\rho)}, \ldots, \frac{1}{\psi_{N-1}^{(N)}(\rho)}\right] W^{-1} .
$$

A direct calculation yields

$$
\left[G^{-1}\right]_{k j}=\frac{1}{N} \sum_{p=1}^{N} \frac{\omega^{(k-j)(p-1)}}{\psi_{p-1}^{(N)}(\rho)} \quad(j, k=1,2, \ldots, N) .
$$

This makes it possible to solve (3.1) for $Q_{k}(k=1,2, \ldots, N)$ as

$$
Q_{k}=\frac{1}{N} \sum_{j, p=1}^{N} \frac{\omega^{(k-1)(p-1)-(j-1)(p-1)}}{\psi_{p-1}^{(N)}(\rho)} f\left(z_{j}\right),
$$

hence we have

$$
f^{(N)}(z)=\sum_{n=0}^{\infty} b_{-n} \rho^{-n} \frac{\psi_{-n}^{(N)}(z)}{\psi_{-n}^{(N)}(\rho)}
$$


Consequently we can write the error function $e^{(N)}=f-f^{(N)}$ as

$$
e^{(N)}(z)=\sum_{n=0}^{\infty} b_{-n} \rho^{-n}\left[\left(\frac{\rho}{z}\right)^{n}-\frac{\psi_{-n}^{(N)}(z)}{\psi_{-n}^{(N)}(\rho)}\right] .
$$

We obtain by the maximum modulus principle for holomorphic functions

$$
\sup _{z \in \bar{\Omega}}\left|e^{(N)}(z)\right| \leq \sum_{n=0}^{\infty}\left|b_{-n}\right| \rho^{-n} g_{n, \rho}^{(N)},
$$

where

$$
g_{n, \rho}^{(N)}:=\sup _{z \in \gamma_{\rho}}\left|\left(\frac{\rho}{z}\right)^{n}-\frac{\psi_{-n}^{(N)}(z)}{\psi_{-n}^{(N)}(\rho)}\right| .
$$

At this stage we claim the following Lemma 3.4 concerning $g_{n, \rho}^{(N)}$.

LEMmA 3.4. (i) For all $n \in \mathbb{N} \cup\{0\}$, we have

$$
g_{n, \rho}^{(N)} \leq 2 .
$$

(ii) For all $p \in\{0,1, \ldots, N-1\}$, we have

$$
g_{p, \rho}^{(N)} \leq \frac{2(R / \rho)^{N}}{1-(R / \rho)^{N}} .
$$

By (3.8) and Lemma 3.4, we can give a proof of Theorem 2.4.

\section{Condition Numbers}

In this section we calculate the condition number of the coefficient matrix $G$ for the linear system (3.1).

(I) In the case $\Omega=D_{\rho}$. Since the coefficient matrix $G$ is an Hermitian matrix, if we take a norm for $N$-dimensional vectors as

$$
\|\boldsymbol{x}\|:=\sqrt{\sum_{j=1}^{N}\left|x_{j}\right|^{2}} \quad\left(\boldsymbol{x}=\left(x_{1}, x_{2}, \ldots, x_{N}\right)^{\mathrm{T}}\right),
$$

we can calculate the condition number of $G$ as

$$
\text { cond } G=\|G\| \cdot\left\|G^{-1}\right\|=\frac{\max _{p=1,2, \ldots, N}\left|\varphi_{p}^{(N)}(\rho)\right|}{\min _{p=1,2, \ldots, N}\left|\varphi_{p}^{(N)}(\rho)\right|}=\frac{\left|\varphi_{1}^{(N)}(\rho)\right|}{\left|\varphi_{N}^{(N)}(\rho)\right|}=\left(\frac{R}{\rho}\right)^{N-1} \text {. }
$$


(II) In the case $\Omega=D_{\rho}^{*}$. We can calculate cond $G$ similarly in the case (I) as

$$
\text { cond } G=\left(\frac{\rho}{R}\right)^{N-1} \text {. }
$$

We know from (I) and (II) that cond $G$ increases exponentially with respect to $N$, therefore the linear system (3.1) is ill-conditioned. Nevertheless, as we shall see in Section 5, numerical computations perform well.

On the other hand, in CSM, cond $G=O\left((R / \rho)^{N / 2}\right)$ or $O\left((\rho / R)^{N / 2}\right)$ in the case $\Omega=$ $D_{\rho}$ or $\Omega=D_{\rho}^{*}$, respectively, therefore the linear system for $Q_{k}$ 's is ill-conditioned, too. There are results about the numerical stability of CSM by Kitagawa [4, 5]. Studying the numerical stability of CDSM will be expected, too.

\section{Numerical Experiments}

In this section we introduce results for our numerical experiments in the case $\Omega=$ $D_{\rho}$. Errors of CSM and DSM show almost similar behavior, thus we show results for DSM, and compare it with that for CDSM. A corresponding Dirichlet problem which is solved numerically by DSM is as follows:

$$
\begin{aligned}
\triangle u & =0 \quad \text { in } \Omega, \\
u=\operatorname{Re} f & \text { on } \partial \Omega .
\end{aligned}
$$

The collocation points $\left\{z_{j}\right\}_{j=1}^{N}$ and the charge points $\left\{\zeta_{k}\right\}_{k=1}^{N}$ are defined by

$$
z_{j}=\rho \omega^{j-1} \quad(j=1,2, \ldots, N) \quad \text { and } \quad \zeta_{k}=R \omega^{k-1} \quad(k=1,2, \ldots, N),
$$

where $R$ is a parameter which satisfies $R>\rho$. In order to estimate numerically the error $\sup _{z \in \bar{\Omega}}\left|f(z)-f^{(N)}(z)\right|$, we use the Monte Carlo method. In other words, we adopt the following quantity as an approximation of the error:

$$
\widetilde{e}^{(N)}:=\max _{z \in \Lambda}\left|f(z)-f^{(N)}(z)\right|,
$$

where $\Lambda$ is a set of points chosen from $\gamma_{\rho}$. To be more concrete, we prepare a set $\Theta:=$ $\left\{\theta_{k}\right\}_{k=1}^{M} \subset[0,1[(M$ is equal to $10 \cdot \max N)$ by using pseudo-random numbers, and put

$$
\Lambda:=\left\{\rho \mathrm{e}^{2 \pi \mathrm{i} \theta} ; \theta \in \Theta\right\} .
$$

Likewise, we take up

$$
\widetilde{e}_{\mathrm{D}}^{(N)}:=\max _{z \in \Lambda}\left|u(z)-u_{\mathrm{D}}^{(N)}(z)\right|
$$

as an approximation of the error for DSM. Test 1, 2-(i), 3 and 4-(i) were performed by using MATLAB, and Test 2-(ii) and 4-(ii) by $\mathrm{C}++$ programs using the multiple-precision arithmetic library "exflib" (see Fujiwara [1]). 


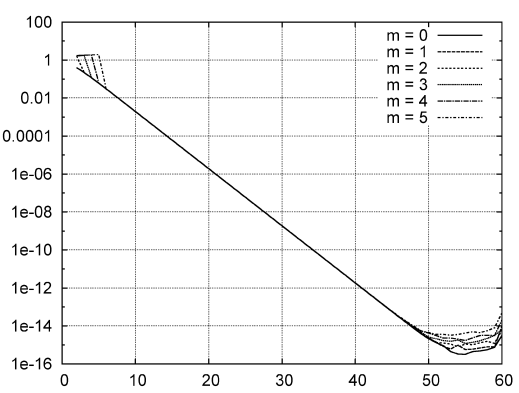

(a) CDSM

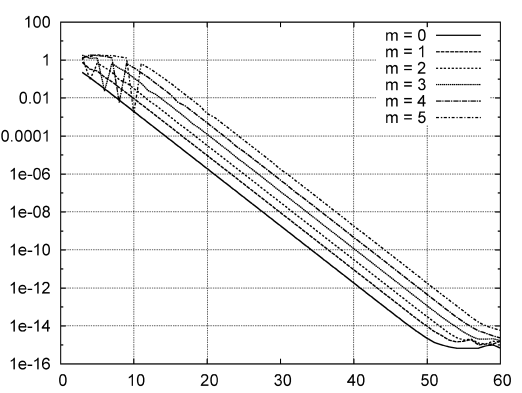

(b) DSM

FIGURE 1. $f(z)=z^{m}$, where $m \in\{0,1, \ldots, 5\}, N \in\{2,3, \ldots, 60\}, \rho=1$ and $R=2$

In each numerical experiment, we compute $\widetilde{e}^{(N)}$ and $\widetilde{e}_{\mathrm{D}}^{(N)}$ and plot graphs whose horizontal axis represents $N$ and vertical axis $\log _{10} \widetilde{e}^{(N)}$ or $\log _{10} \widetilde{e}_{\mathrm{D}}^{(N)}$.

Test 1. In this test we deal with the case where $f$ is a power function whose exponent is a non-negative integer:

$$
f(z)=z^{m}
$$

where $m \in\{0,1, \ldots, 5\}$ is a parameter.

In Figure 1, we find these graphs are almost linear. This means that $\widetilde{e}^{(N)}$ and $\widetilde{e}_{\mathrm{D}}^{(N)}$ behave as

$$
\widetilde{e}^{(N)} \sim C \tau^{N}, \quad \widetilde{e}_{\mathrm{D}}^{(N)} \sim C^{\prime} \tau^{\prime N} \quad(N \rightarrow \infty) .
$$

We know that $\tau$ and $\tau^{\prime}$ are almost equal to $\rho / R$ in this case since $f$ is holomorphic and $u$ harmonic in the entire plane, so that suprema of $r_{0}$ 's which satisfy the condition of Theorems 2.2 and 1.1 , respectively, are equal to $+\infty$.

Test 2. In this test we deal with the case where $f$ is an exponential function composed with a power function whose exponent is a non-negative integer:

$$
f(z)=\exp \left(z^{m}\right)
$$

where $m \in\{0,1, \ldots, 5\}$ is a parameter.

(i) We compute $\widetilde{e}^{(N)}$ and $\widetilde{e}_{\mathrm{D}}^{(N)}$ for all $N \in\{2,3, \ldots, 60\}$ when $(\rho, R)=(1,2)$, and $N \in\{2,3, \ldots, 100\}$ when $(\rho, R)=(1,1.5)$ (see Figure 2$)$.

Since $f$ is holomorphic and $u$ harmonic in the entire plane, $\tau$ and $\tau^{\prime}$ are both almost equal to $\rho / R$ as well as Test 1 , however, we cannot see from Figures 2 (a) and (b) that $\widetilde{e}^{(N)}$ and $\widetilde{e}_{\mathrm{D}}^{(N)}$ behave as (5.1). On the other hand, from Figure 2 (c), $\widetilde{e}^{(N)}$ behaves as (5.1) for $m \in$ $\{0,1,2,3,4\}$. Simultaneously, from Figure $2(\mathrm{~d}), \widetilde{e}_{\mathrm{D}}^{(N)}$ behaves as (5.1) for $m \in\{0,1,2,3\}$. 


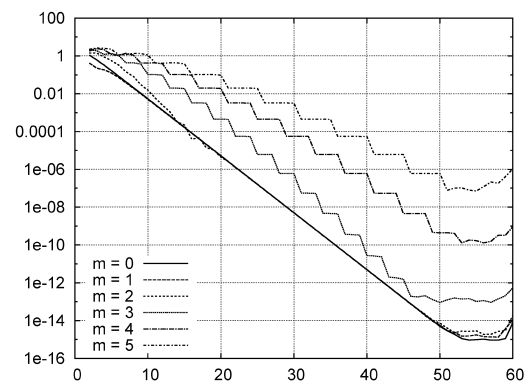

(a) CDSM

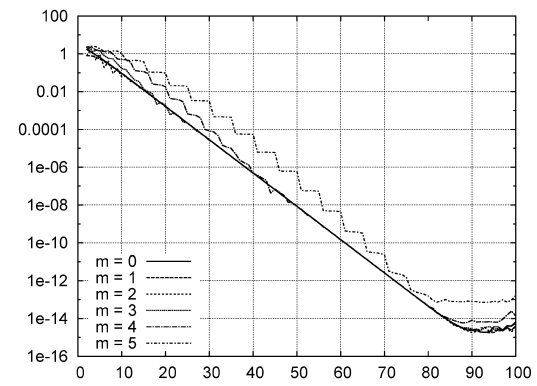

(c) CDSM

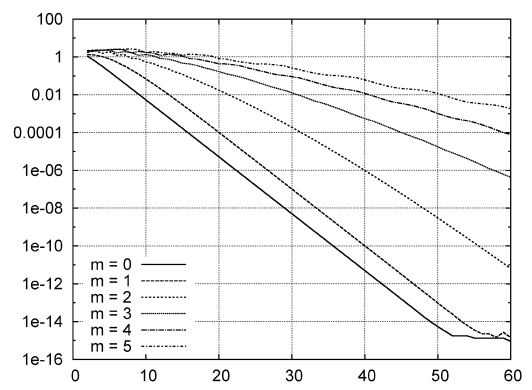

(b) DSM

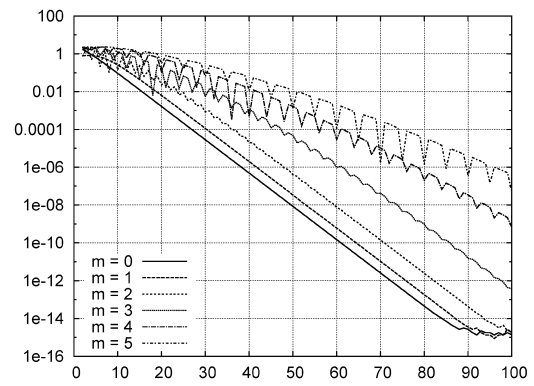

(d) DSM

FIGURE 2. $f(z)=\exp z^{m}$ where $m \in\{0,1, \ldots, 5\}$, in double-precision: (a), (b) $N \in\{2,3, \ldots, 60\}$, $\rho=1$ and $R=2 ;$ (c), (d) $N \in\{2,3, \ldots, 100\}, \rho=1$ and $R=1.5$

Hence we can investigate that $\widetilde{e}^{(N)}$ and $\widetilde{e}_{\mathrm{D}}^{(N)}$ behave as (5.1) for sufficiently large $N$.

In order to clear up this investigation, we compute $\widetilde{e}^{(N)}$ with $(\rho, R)=(1,2)$ and $\widetilde{e}_{\mathrm{D}}^{(N)}$ with $(\rho, R)=(1,1.5)$ for larger $N$ using multiple-precision arithmetic.

(ii) We compute $\widetilde{e}^{(N)}$ and $\widetilde{e}_{\mathrm{D}}^{(N)}$ for all $N \in\{2,3, \ldots, 400\}$ in 120 digits precision by $\mathrm{C}++$ programs using exflib (see Figure 3 ).

We can see from Figure 3 that $\widetilde{e}^{(N)}$ and $\widetilde{e}_{\mathrm{D}}^{(N)}$ behave as (5.1).

Test 3. In this test we deal with the case where $f$ is a rational function:

$$
f(z)=\frac{1}{z-p(m)}, \quad p(m)=\rho+0.1+0.2 m,
$$

where $m \in\{0,1, \ldots, 10\}$ is a parameter. $f$ has a simple pole at $p(m)$.

We find $\widetilde{e}^{(N)}$ and $\widetilde{e}_{\mathrm{D}}^{(N)}$ behave as (5.1), and since suprema of $r_{0}$ 's satisfying the condition of Theorems 2.2 and 1.1 are both equal to $p(m)$, so that $\tau$ and $\tau^{\prime}$ are equal to $\max \{\rho / R, \rho / p(m)\}$ and $\max \{\rho / R, \sqrt{\rho / p(m)}\}$, respectively.

Test 4. In this test we deal with the case where $f$ is an exponential function composed 


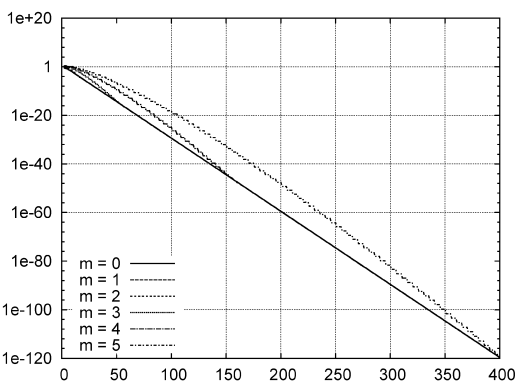

(a) CDSM

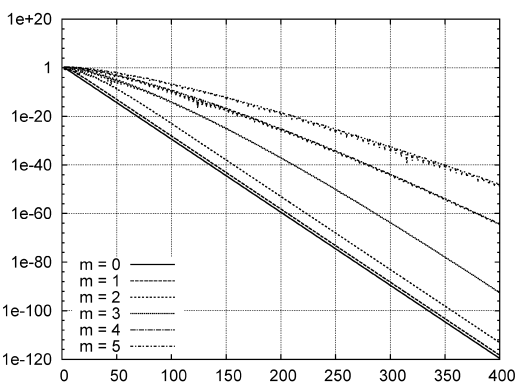

(b) DSM

FIGURE 3. $f(z)=\exp z^{m}$ where $m \in\{0,1, \ldots, 5\}, N \in\{2,3, \ldots, 400\}$, in multiple-precision (120 digits): (a) $\rho=1$ and $R=2$; (b) $\rho=1$ and $R=1.5$

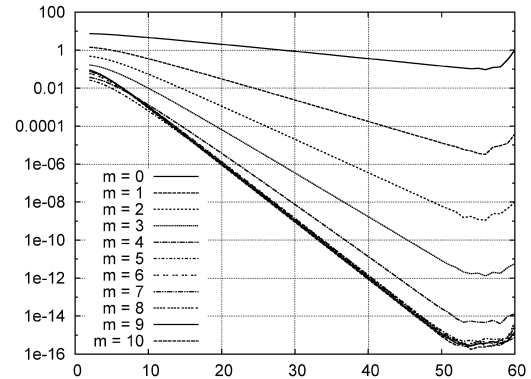

(a) CDSM

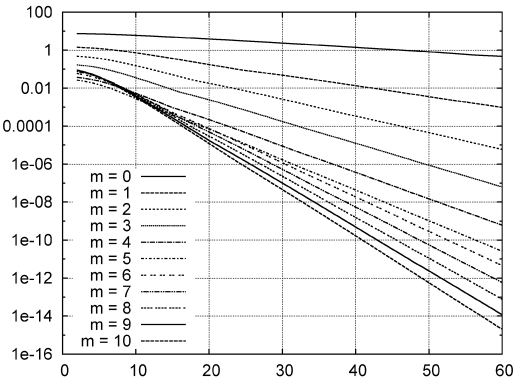

(b) DSM

FIGURE 4. $f(z)=1 /(z-p(m))$ where $m \in\{0,1, \ldots, 10\}, N \in\{2,3, \ldots, 60\}, \rho=1$ and $R=2$

with a rational function:

$$
f(z)=\exp \left(\frac{1}{z-p(m)}\right), \quad p(m)=\rho+0.1+0.2 m,
$$

where $m \in\{0,1, \ldots, 10\}$ is a parameter. $f$ has an essential singularity at $p(m)$.

(i) We compute $\widetilde{e}^{(N)}$ and $\widetilde{e}_{\mathrm{D}}^{(N)}$ for all $N \in\{2,3, \ldots, 60\}$.

(ii) In order to elucidate the same investigation of Test 2 , we compute $\widetilde{e}^{(N)}$ and $\widetilde{e}_{\mathrm{D}}^{(N)}$ for all $N \in\{2,3, \ldots, 400\}$ in 120 digits precision by $\mathrm{C}++$ programs using exflib.

We find from Figure 6 that $\widetilde{e}^{(N)}$ and $\widetilde{e}_{\mathrm{D}}^{(N)}$ behave as (5.1), and $\tau$ and $\tau^{\prime}$ are equal to $\max \{\rho / R, \rho / p(m)\}$ and $\max \{\rho / R, \sqrt{\rho / p(m)}\}$, respectively, in this case by the same reason for Test 3 .

We summarize the results of our numerical experiments. 


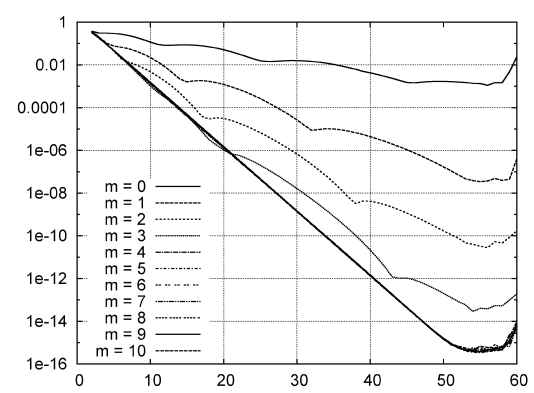

(a) CDSM

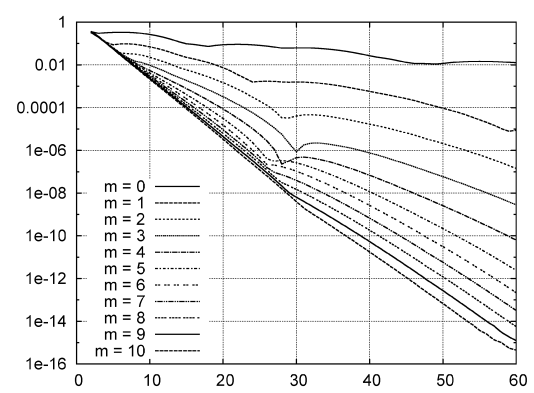

(b) DSM

FIGURE 5. $f(z)=\exp (1 /(z-p(m)))$ where $m \in\{0,1, \ldots, 10\}, N \in\{2,3, \ldots, 60\}$, in double-precision: $\rho=1$ and $R=2$

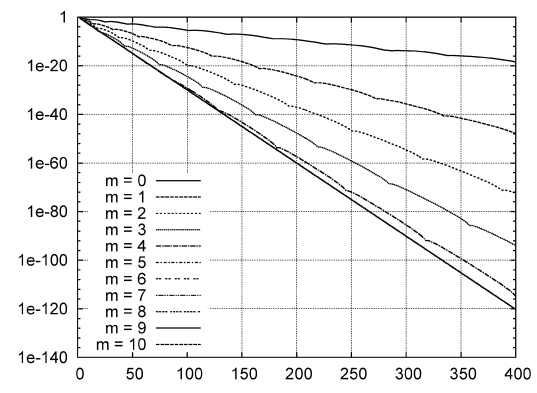

(a) CDSM

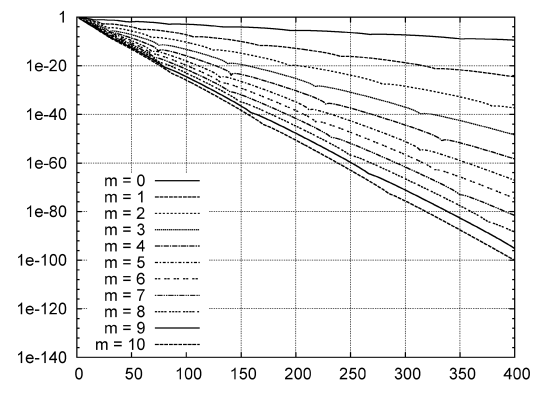

(b) DSM

FIGURE 6. $f(z)=\exp (1 /(z-p(m)))$ where $m \in\{0,1, \ldots, 10\}, N \in\{2,3, \ldots, 400\}$, in multiple-precision (120 digits), $\rho=1$ and $R=2$

- The behaviors of $\widetilde{e}^{(N)}$ and $\widetilde{e}_{\mathrm{D}}^{(N)}$ are what we can expect from the error estimates (see Theorems 2.2 and 1.1).

- $\widetilde{e}^{(N)}$ decays exponentially with respect to $N$, and the gradient of the straight section of $N-\log _{10} \widetilde{e}^{(N)}$ curve is almost equal to

$$
\max \left\{\log _{10} \frac{\rho}{R}, \log _{10} \frac{\rho}{r_{0}}\right\} .
$$

Simultaneously, $\widetilde{e}_{\mathrm{D}}^{(N)}$ decays exponentially with respect to $N$, and the gradient of the 
straight section of $N-\log _{10} \widetilde{e}_{\mathrm{D}}^{(N)}$ curve is nigh equal to

$$
\max \left\{\log _{10} \frac{\rho}{R}, \frac{1}{2} \log _{10} \frac{\rho}{r_{0}}\right\} .
$$

- Best selection of $R$ 's for CDSM and DSM is $R=r_{0}$ and $R=\sqrt{\rho r_{0}}$ (the geometric mean of $\rho$ and $r_{0}$ ), respectively, hence the fastest decaying speed of the error for CDSM and DSM is $\left(\rho / r_{0}\right)^{N}$ and $\left(\rho / r_{0}\right)^{N / 2}$, respectively. In the light of this fact, the convergence rate for CDSM is faster than that for DSM, and to incarnate it, we have to choose largish $R$, but we can achieve the smallest error for smaller $N$.

\section{Concluding Remarks}

In the previous sections, we proposed CDSM which approximates a holomorphic function $f$ by a linear combination of $1 /(z-\zeta)$ of the form (1.4) using boundary values of $f$. A theoretical analysis shows the exponential convergence of the approximate function which is constructed by CDSM, and the result of our numerical experiments exemplify the effectiveness of CDSM.

The results of this paper deal with the case where $\Omega$ is a disk or the exterior domain of a disk. As a matter of fact, we could prove the unique existence and convergence of the approximate function in the case where $\Omega$ is an annular domain and an elliptic domain. We will exhibit these results in another paper.

\section{References}

[ 1 ] H. FUJIWARA, http://www-an.acs.i.kyoto-u.ac.jp/ fujiwara/exflib/

[2] M. Katsurada and H. Окамото, A mathematical study of the charge simulation method I, J. Fac. Sci. Univ. Tokyo Sect. IA Math. 35 (1988), no. 3, 507-518.

[ 3 ] M. Katsurada, A mathematical study of the charge simulation method II, J. Fac. Sci. Univ. Tokyo Sect. IA Math. 36 (1989), no. 1, 135-162.

[4] T. Kitagawa, On the numerical stability of the method of fundamental solution applied to the Dirichlet problem, Japan J. Appl. Math. 5 (1988), no. 1, 123-133.

[ 5 ] T. Kitagawa, Asymptotic stability of the fundamental solution method, Proceedings of the International Symposium on Computational Mathematics (Matsuyama, 1990), J. Comput. Appl. Math. 38 (1991), no. 13, 263-269.

[6] H. OGATA, The dipole simulation method and its application to the numerical conformal mapping (in Japanese), Sūrikaisekikenkyūsho Kōkyūroku, No. 1791 (2012), 97-106.

[ 7 ] H. OGATA, Dipole simulation method for two-dimensional potential problems, NOLTA, IEICE 5 (2014), no. 1, $2-14$. 
Present Addresses:

KOYA SAKAKIBARA

Graduate School of Mathematical Sciences,

THE UNIVERSITY OF TOKYO,

3-8-1 Komaba, Meguro-Ku, TOKYo 153-8914, JAPAN.

e-mail: ksakaki@ms.u-tokyo.ac.jp

MASASHI KATSURADA

SCHOOL OF INTERDISCIPLINARY MATHEMATICAL SCIENCES, MEIJI UNIVERSITY,

4-21-1 NAKANO, NAKANO-KU, TOKYO 164-8525, JAPAN.

e-mail:katurada@meiji.ac.jp 